\title{
COMPLEXITY OF CARDIAC AUTONOMIC MODULATION IN DIABETES MELLITUS: A NEW TECHNIQUE TO PERCEIVE AUTONOMIC DYSFUNCTION
}

\author{
David M. Garner ${ }^{1,}$, Naiara Maria de Souza ${ }^{2}$, Vitor E. Valenti ${ }^{3}$, Luiz Carlos M. Vanderlei ${ }^{2}$ \\ ${ }^{1}$ Cardiorespiratory Research Group, Department of Biological and Medical Sciences, \\ Faculty of Health and Life Sciences, Oxford Brookes University, Headington Campus, \\ Gipsy Lane, Oxford OX3 0BP, Oxfordshire, United Kingdom \\ ${ }^{2}$ Department of Physiotherapy, Sao Paulo State University, UNESP, Presidente \\ Prudente, SP, Brazil \\ 3 Autonomic Nervous System Center, Sao Paulo State University, UNESP, Marília, SP, \\ Brazil

$$
\text { received: }
$$$$
\text { August 22, } 2019
$$$$
\text { accepted: }
$$$$
\text { August 29, } 2019
$$$$
\text { available online: }
$$$$
\text { October 21, } 2019
$$

\begin{abstract}
Backgound and aims. In this study we analyzed heart rate variability (HRV) via chaotic global techniques so as to discriminate diabetics from control subjects. Matherial and method. Chaotic global analysis of the RR-intervals from the electrocardiogram and preprocessing adjustments were undertaken. The effect of varying two parameters to adjust the Multi-Taper Method (MTM) power spectrum were evaluated. Then, cubic spline interpolations from $1 \mathrm{~Hz}$ to $13 \mathrm{~Hz}$ were applied whilst the spectral parameters were fixed. Precisely 1000 RR-intervals of data were recorded. Results. CFP1 and CFP3 are the only significant combinations of chaotic globals when the default standard conditions are enforced. MTM spectral adjustments and cubic spline interpolation are trivial at effecting the outcome between the two datasets. The most influencial constraint on the outcome is data length. Conclusion. Chaotic global analysis was offered as a reliable, low-cost and robust technique to detect autonomic dysfunction in subjects with diabetes mellitus.
\end{abstract}

key words: Diabetes Mellitus; Multi-Taper Method; Cubic Spline Interpolation; DPSS; Thomson's nonlinear combination

\section{Background and Aims}

Heart rhythm is one of the key signs for distinguishing a pathological state. Since heart rate presents short term variability, mathematical algorithms to analyze these oscillations are necessary to develop novel methods to recognize cardiovascular diseases early and achieve differential diagnosis [1].
The rhythm of electrocardiographic (ECG) RR-intervals resulting from the PQRSTsignature can waver in a chaotic manner $[\underline{2}, \underline{3}]$. Currently, we aim to evaluate the cardiac branch of the autonomic nervous system (ANS) of the subjects with diabetes mellitus by analyzing their heart rate variability (HRV) [4]. Such computations are beneficial to assess surgical patients [] particularly when sedated [] or

Oxford Brookes University, Headington Campus, Gipsy Lane, Oxford OX3 0BP, Oxfordshire, United Kingdom. corresponding author e-mail: davidmgarner1@gmail.com 
unable to indicate discomfort as with sleep apnea patients [7] or those experiencing "air hunger" $[\underline{8}, \underline{9}]$. Thus, the method advises the clinical team to identify delicate changes in the ANS, in addition to predicting the risk of difficulties.

The unit necessary for HRV analysis are the RR-intervals and, physiological mechanisms related to heart rate control are nonlinear [1]. Through RR-intervals we compute the innovative chaotic global parameters to determine the control from the experimental time-series. We expected the subjects with diabetes mellitus' RR-intervals chaotic responses performed in a nonlinear manner equivalent to cardiac arrest $[\underline{10}, \underline{11}]$, during epileptic seizures $[\underline{12}, 13]$, chronic obstructive pulmonary disease (COPD) [14] and attention deficit hyperactivity disorder (ADHD) [15] when computed using these algorithms.

A lessening in the levels of chaos would be consistent with abnormalities in the ANS and a dysfunctional vagus. This nerve has a significant role in regulation of the rhythm in physiological systems [16]. The sympathetic and parasympathetic nervous systems' connections have been shown to influence HRV. HRV is a non-invasive and inexpensive tool to monitor the cardiac branches of the ANS. Further techniques are insensitive for example the Sympathetic Skin Response [17] or, too costly such as Quantitative Pupillography [18].

These chaotic global techniques are highly sensitive to such variabilities. This is particularly the case compared with those based on linear descriptive statistics, conventional nonlinear or geometric routines. The greater the levels of chaos, usually the more healthy the physiological status. Less chaos can be interpreted as a statistical marker for pathological states and "dynamical diseases" in particular [19].
Historically, we have calculated HRV in

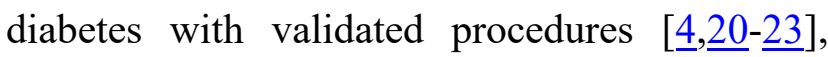
yet, the reliability of global chaotic analysis to detect autonomic impairment in diabetic patients is unclear. Reduced chaos is related to decreased HRV and impaired physiological status [19,24]. Our initial hypothesis proposed that diabetic patients presented reduced chaotic behavior of HRV. In this circumstance, we evaluated HRV through chaotic global techniques with the intention of discriminating diabetics from control subjects and confirm its reliability to identify ANS dysfunction.

\section{Material and Methods}

Patient Selection and assessments were exactly as the studies by Souza et al [22] and Garner et al [4].

\section{Multi-Taper Method (MTM) \\ Power Spectrum}

MTM is a power spectrum that can be advantageous when applied in spectral estimation [4]. Its key advantage is minimization of spectral leakage. Functions named discrete prolate spheroidal sequences (DPSS); often termed Slepian Sequences [25] are a set of functions which maximize the windows. See Figure 1.

\section{Chaotic Globals}

High spectral Entropy (hsEntropy) [4] is a computation based on the unevenness of the amplitude and frequency of the MTM power spectrums peaks. Shannon entropy [26] is the function applied to the power spectrum. We compute the median Shannon entropy of three values attained from three various MTM power spectra. Thus, the MTM power spectra at three test settings: (a) a sine wave, (b) uniformly distributed random variables, and (c) the oscillating signal from the subject with diabetes mellitus. These values are normalized. The sine 
wave achieves a zero value, the uniformly $h s$ Entropy is this corresponding 'in between' random variable attains unity, and the diabetic value. subjects' experimental signal between.

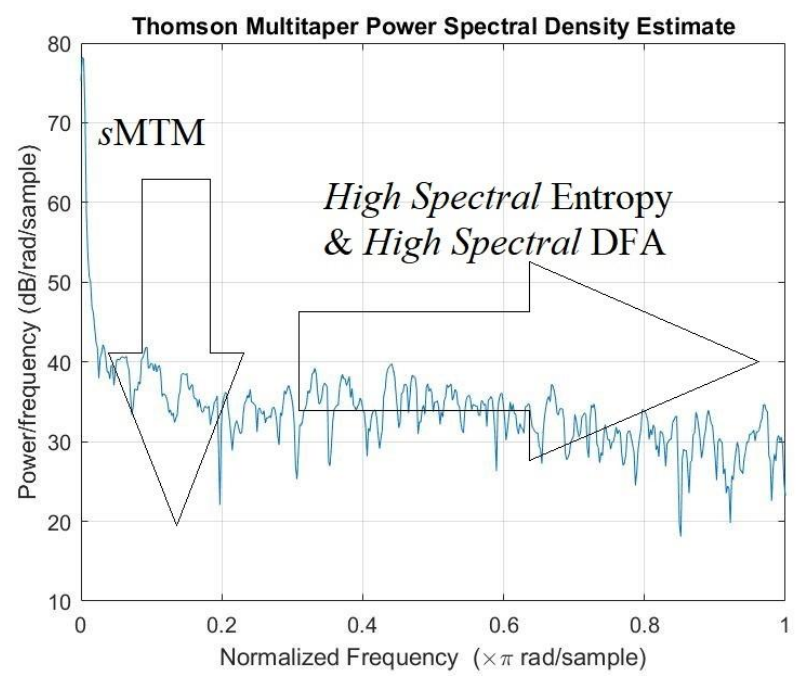

Figure 1. An MTM power spectrum for an arrangement of 1000 RR-intervals from a subject diagnosed with diabetes mellitus. sMTM is the area underneath the power spectrum; yet above the baseline. MTM parameters used in the computation of sMTM, high spectral Entropy and high spectral DFA are as follows (a) $1 \mathrm{~Hz}$ for sampling frequency; (b) DPSS or Slepian Sequences are set to 3; (c) 256 for Fast Fourier Tranform length; and, lastly (d) Thomson's nonlinear combination method is set to 'adaptive'.

DFA was derived by Peng et al in 1995 [27]. It can be imposed on time-series where the mean, variance and autocorrelation adjust with time. High spectral Detrended Fluctuation Analysis ( $h s$ DFA) is where DFA is applied to the frequency rather than time. The horizontal axis is frequency and, vertical axis is amplitude. To acquire $h s$ DFA we estimate the spectral adaptation in precisely the same way as with $h s$ Entropy. DFA is the algorithm enforced onto the power spectrum instead.

Spectral Multi-Taper Method (sMTM) [28] is derived from elevated broadband noise intensities generated in MTM power spectra by irregular and often chaotic signals. SMTM is the area beneath the power spectrum but above the baseline.

\section{Chaotic Forward Parameters (CFP 1 to CFP7)}

CFP1 to CFP7 [4] are applied to RRintervals from normal subjects and those with diabetes mellitus. $h s$ DFA responds to chaos contrariwise to the others, so we subtract its value from unity. Weightings of unity are assumed for all three chaotic globals. Later we evaluate the statistically significant CFP1 and CFP3 alone. We applied the CFP1 with all three chaotic globals included and then the CFP3 with $h s$ DFA absent. As before in Souza et al [22] and Garner et al [4], CFP1 is expected to be the most statistically robust and the CFP3 the most statistically significant.

There are seven non-trivial combinations of three chaotic globals [15]. It is anticipated that the CFP which applies all three should be the most robust. This is since it takes the information and processes it in three different ways. The summation of the three would be expected to deviate greater than single or double permutations. The potential analytical hazard here is we are only computing spectral components; the phase information is lost. 


\section{Principal Component Analysis}

Principal Component Analysis (PCA) $[\underline{29}, \underline{30}]$ estimates the complexity of highdimensional data sets. It is sympathetic when sources of unpredictability in the data need clarification or, to lessen the complexity of the data and, via this evaluate the data with fewer dimensions. Its key aim is to signify the data with least variables at the same time as satisfying the majority of total variance.

There are two main properties of the PCA:

- It is non-parametric - no prior knowledge can be incorporated.

- Data reduction incurs loss of information.

Some assumptions of the technique are imperative:

1. Linearity - Accepting the data set to be linear combinations of the variables.

2. The necessity of mean and covariance.

3. No assurance that the direction of maximum variance will contain discriminative features.

4. Large variance includes the most important dynamics, whilst the lowest corresponds to noise.

The following need consideration when understanding PCA:

1. The higher the component loadings the more critical that the variable is to the component.

2. Positive and negative loadings are understood as mixed.

3. Whether positive or negative for mixed loadings; it is irrelevant.

4. The rotated component matrix is vital.

\section{Effect Sizes}

Cohen's $d[\underline{31}, \underline{32}]$ is a general term that signifies the effect sizes. To estimate the size of deviations amongst protocols for significant differences, the effect size was assessed via a sub-group termed Cohen's $d_{\mathrm{s}}$ [33]. Cohen's $d_{s}$ characterize the standardized mean difference of an effect. It can be conferred to compute effects across studies even when the dependent variables are quantified in unalike ways or when completely dissimilar measures are affected. It varies from zero to infinity and can be positive or negative. Cohen denotes the standardized mean difference between two groups of independent observations for a suitable sample as $d_{\mathrm{s}}$.

So for Cohen's $d_{s}$ (equation below), the numerator is the variation between the means of two groups of observations. The denominator is the pooled standard deviation. These are then squared to avert the positive and negative values cancelling each other out. They are summed, and divided by the number of observations minus one (Bessel's correction) for bias in the estimate of the population variance. To conclude, a square root is performed.

$$
\text { Cohen's } d_{s}=\frac{\bar{X}_{1}-\bar{X}_{2}}{\sqrt{\frac{\left(n_{1}-1\right) S D_{1}^{2}+\left(n_{2}-1\right) S D_{2}^{2}}{n_{1}+n_{2}-2}}}
$$

Regarding, Cohen's $d_{s}$ the subsequent values describe their relevance as per Sawilowsky [34]; $0.01>$ very small effect; $0.20>$ small effect; $0.50>$ medium effect; $0.80>$ large effect; $1.20>$ very large effect, and finally $2.00>$ a huge effect size.

\section{Thomson's nonlinear combination methods \& DPSS}

Now we assess the effect of manipulating Thomson's nonlinear combination settings on the MTM spectra. There are three possible circumstances. The conditions that can be imposed on the MTM power spectrums window are 'adapt', 'eigen', or 'unity' and are the weights on individual tapered power spectral density (PSD) estimates. The default is Thomson's 
adaptive frequency-dependent weights, hence 'adapt'. The 'eigen' method weights each tapered PSD estimate by the eigenvalue (frequency concentration) of the corresponding Slepian taper. The 'unity' method weights each tapered PSD estimate equally [21].

Moreover, we concurrently assess the effect of changing the settings of the DPSS from 2 to 13. DPSS affects the adaptation properties of the tapers with the intention of reducing spectral leakage. Whilst assessing the outcomes of the Thomson's nonlinear combinations settings and the levels of DPSS on the chaotic response the sampling frequency is fixed at $1 \mathrm{~Hz}$ for the MTM and Fast Fourier Transform of length 256 is enforced. We assess the effects of DPSS (2 to 13) and Thomson's nonlinear combinations ('adaptive','eigen' and 'unity') at multiple lengths of time-series of RR-intervals simultaneously. We start the assessment at 500 RR-intervals and at intervals of 50 increase the time-series to a maximum of $1000 \mathrm{RR}$-intervals. We only assess CFP1 and CFP3. These are the only groupings significant under default conditions.

\section{Cubic-spline Interpolation}

Following this, we evaluated the importance of pre-processing techniques on the results obtained through chaotic global algorithms. Once more comparing only the chaotic global values for CFP1 and CFP3 as these are the only values significant under default conditions.

Time series constructed from the RRinterval tachograms are not equidistantly sampled. This has to be made suitable prior to frequency-domain analysis. Firstly, we can decide to assume equidistant sampling [35] and compute the power spectrum directly from the tachogram of RR-intervals. This is the method widely adopted up-til-now by previous studies on chaotic globals with obese children [36],
ADHD [15] and diabetes mellitus [22]. The RRintervals are therefore a function of the beat number. Yet, this could cause a distortion in the spectrum [37] and so the spectrum must then be considered a function of cycles per beat rather than of frequency [38].

An alternative approach is to implement a cubic spline interpolation [39] to convert the nonequidistantly sampled RR-tachogram into an equidistantly sampled time series [40]. Consequently we arranged a cubic spline interpolation on the RR-interval tachogram. We accomplish this at the levels $1 \mathrm{~Hz}$ to $13 \mathrm{~Hz}$. This covers the most relevant scenarios in HRV analysis. Kubios $\mathrm{HRV}^{\circledR}$ [23] software offers a default option of $4 \mathrm{~Hz}$. It is important to realize that the interpolation frequency will increase the number of data points in the time-series. A frequency of $4 \mathrm{~Hz}$ for example will elevate the number of RR-intervals from 1000 to 4000 , etc.

Following the interpolation, the chaotic global algorithms are fixed. During the assessment of the cubic spline interpolations we set the Thomson's setting to 'adaptive', $1 \mathrm{~Hz}$ for sampling frequency, length of 256 for Fast Fourier Transform and the DPSS set to 3 .

\section{Results}

When observing Table 1 it should be emphasised that CFP1, CFP2, CFP3, CFP6 and CFP7 present increased values in the normal physiological healthy state and reduced values in the diabetic group. Of the seven permutations only CFP1 and CFP3 are statistically significant with CFP1 $(<0.03$; Kruskal-Wallis, medium effect size) and CFP3 $(<0.01$; ANOVA1 \& Kruskal-Wallis, large effect size). The standard deviations are comparable throughout.

With regards to the multivariate analysis by PCA (See Figure 2). For the MTM power spectra CFP1 has the First Principal Component (PC1) (0.257) and the Second Principal 
Component (PC2) (-0.518). Nevertheless, CFP3 has the PC1 (0.049) and the PC2 (-0.609). Only the first two components need be considered owing to the steep scree plot. The cumulative influence as a percentage is 61.9 percent for the PC1 and 99.8 percent for the cumulative total of the PC1 and PC2. PC2 has an influence of 37.9 percent. CFP1 which applies all three chaotic global techniques is the optimal and most robust overall grouping with regards influencing the correct outcome.

Table 1. Mean values and their standard deviations for the chaotic forward parameters (CFP1 to CFP7: non-dimensional values) for the normal and diabetic mellitus subjects with $1000 \mathrm{RR}$ intervals. Both the one-way analysis of variance (ANOVA1:parametric) and the Kruskal-Wallis (non-parametric) tests of significance were calculated. This was since the normal distributions were borderline in the majority of cases, determined via statistical tests of Anderson-Darling [41] and

Lilliefors [42]. Effect Sizes by Cohen's $d_{s}$ were calculated for CFP1 and CFP3 as they were significant by ANOVA1 and/or Kruskal-Wallis tests under default conditions.

\begin{tabular}{|l|l|l|l|l|l|}
\hline $\begin{array}{l}\text { Chaotic Global } \\
\text { CFPx }\end{array}$ & $\begin{array}{l}\text { Mean } \pm \text { S.D. } \\
\text { Normal }(\mathrm{n}=23)\end{array}$ & $\begin{array}{l}\text { Mean } \pm \text { S.D. } \\
\text { Diabetic }(\mathrm{n}=23)\end{array}$ & $\begin{array}{l}\text { ANOVA1 } \\
(\mathrm{p} \text {-value })\end{array}$ & $\begin{array}{l}\text { Kruskal-Wallis } \\
(\mathrm{p} \text {-value })\end{array}$ & $\begin{array}{l}\text { Cohen's } d_{s} \text { Effect } \\
\text { Sizes }\end{array}$ \\
\hline CFP1 & $0.9217 \pm 0.1194$ & $0.8603 \pm 0.1202$ & 0.0893 & 0.0273 & 0.51 \\
\hline CFP2 & $0.6340 \pm 0.1362$ & $0.5889 \pm 0.0995$ & 0.2066 & 0.1471 & - \\
\hline CFP3 & $0.8467 \pm 0.1072$ & $0.7463 \pm 0.1043$ & 0.0024 & 0.0002 & 0.94 \\
\hline CFP4 & $0.7283 \pm 0.2248$ & $0.7394 \pm 0.1985$ & 0.8597 & 0.8347 & - \\
\hline CFP5 & $0.3268 \pm 0.1725$ & $0.4071 \pm 0.1480$ & 0.0973 & 0.1040 & - \\
\hline CFP6 & $0.6440 \pm 0.1735$ & $0.6133 \pm 0.1504$ & 0.5243 & 0.6445 & - \\
\hline CFP7 & $0.4765 \pm 0.2448$ & $0.3641 \pm 0.1967$ & 0.0931 & 0.1040 & - \\
\hline
\end{tabular}

\section{Component Loadings}

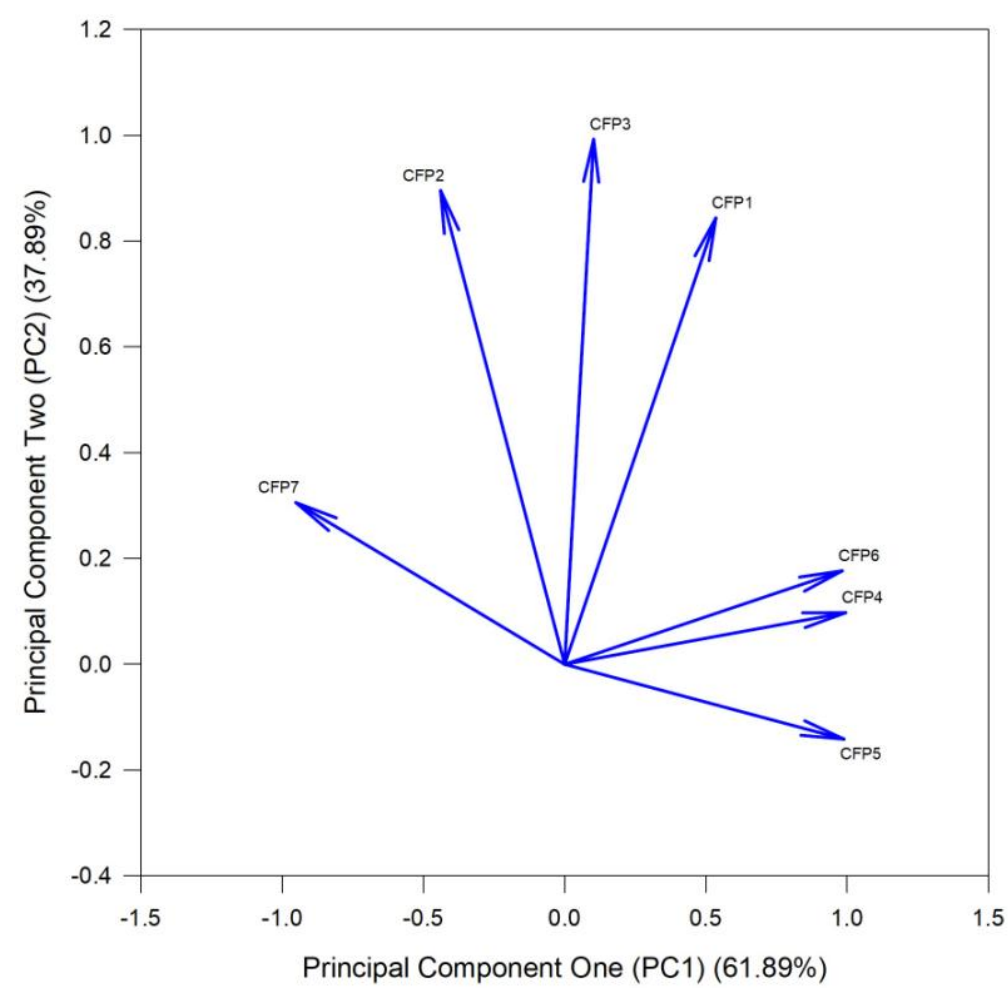

Figure 2. The plot illustrates the component loadings CFP1 to CFP7 for the 1000 RR-intervals of 23 subjects with diabetes mellitus. The CFP values are deduced by using the MTM spectra throughout. The properties of the MTM spectra are as follows: Sampling frequency $1 \mathrm{~Hz}$, DPSS of 3, 256 for Fast Fourier Transform length and Thomson's nonlinear combination set at 'adaptive'. Clearly, CFP1 and CFP3 are the most influencial components when assessed by PCA above. 
Thomson's nonlinear combination methods \& DPSS

Here we present results for CFP1 and CFP3. We chose CFP1 and CFP3 since they are the two combinations presenting significant values under default conditions with MTM power spectra (CFP1 to CFP7) [22]. For CFP1 and CFP3 concerning the Thomson's nonlinear combination methods the difference the three conditions have is minimal. This is also the same regarding the manipulation of the DPSS. Most of the differences are induced by signal length. See sections next on CFP1 and CFP3.

\section{Chaotic Forward Parameter One}

CFP1 (Figure 3): Regarding the DPSS for the 'adapt', 'eigen' and 'unity' settings the decreases were mostly identical throughout. DPSS only effects the p-values on the shortest lengths of data $(<600$ RR-intervals $)$; the interquatile ranges are small at maximal data length (1000 RR-intervals). Next, the KruskalWallis test of significance is more sensitive on the p-values than the ANOVA1 throughout. The p-values on similar datasets are smaller and therefore more significant. Finally, length of the RR-intervals is the most critical factor to attain a statistical marker for dynamical diseases at the level $\mathrm{p}<0.05$ (or $<5 \%$ ) for both the KruskalWallis test and ANOVA1, but only for 900 to 1000 RR-intervals.

\section{Chaotic Forward Parameter Three}

CFP3 (Figure 4): The p-values are more significant for CFP3 compared to CFP1. With CFP3 $p<0.2$ (ANOVA) and $p<0.04$ (KruskalWallis) throughout the analysis. DPSS has greater influence on $\mathrm{p}$-values at lower data lengths than does CFP1. This is revealed by the higher interquartile ranges at low data lengths of the RR-intervals. Note, a length of 600 RRintervals is significant for CFP3 whereas 900
RR-intervals is required for CFP1. There is more statistical variability with CFP3 than with CFP1. Interquartile ranges are higher, boxplot whiskers are wider and the $\mathrm{p}$-values smaller.

\section{Cubic Spline Interpolation}

The effect of cubic spline interpolation between $1 \mathrm{~Hz}$ and $13 \mathrm{~Hz}$ increasing the length of the time-series via interpolation (rather than by recording longer time-series in the laboratory) is minimal. This is for both CFP1 and CFP3; parametric and non-parametric tests of significance as illustrated in Table 2.

\section{Discussions}

It is not totally clear why different algorithms behave in different manner for heart rate autonomic control. Thus, our study aimed to assess a new approach to detect autonomic dysfunction in diabetic patients based on the complexity of RR-intervals oscillation. As a main outcome, chaotic global techniques applied for HRV analysis were able to identify cardiac autonomic dysfunction in a sample of diabetic patients.

HRV has received consideration due to the simple workability of the technique. The data can be collected by a simple one-channel ECG or by a pulse watch. RR-intervals can be processed by user-friendly software [40]. Furthermore, in 1996 the Task Force published directives in order to standardize HRV analysis based on linear methods in the time and frequency domains [40]. In this context, the research literature has established decreased HRV in diabetes $[\underline{43}, \underline{44}]$.

Equally, and more recently, nonlinear analysis of HRV was suggested to provide information related to the scaling, quality and correlation properties of the time series, while traditional linear methods were designed to assess the magnitude of HRV. Complexity of 

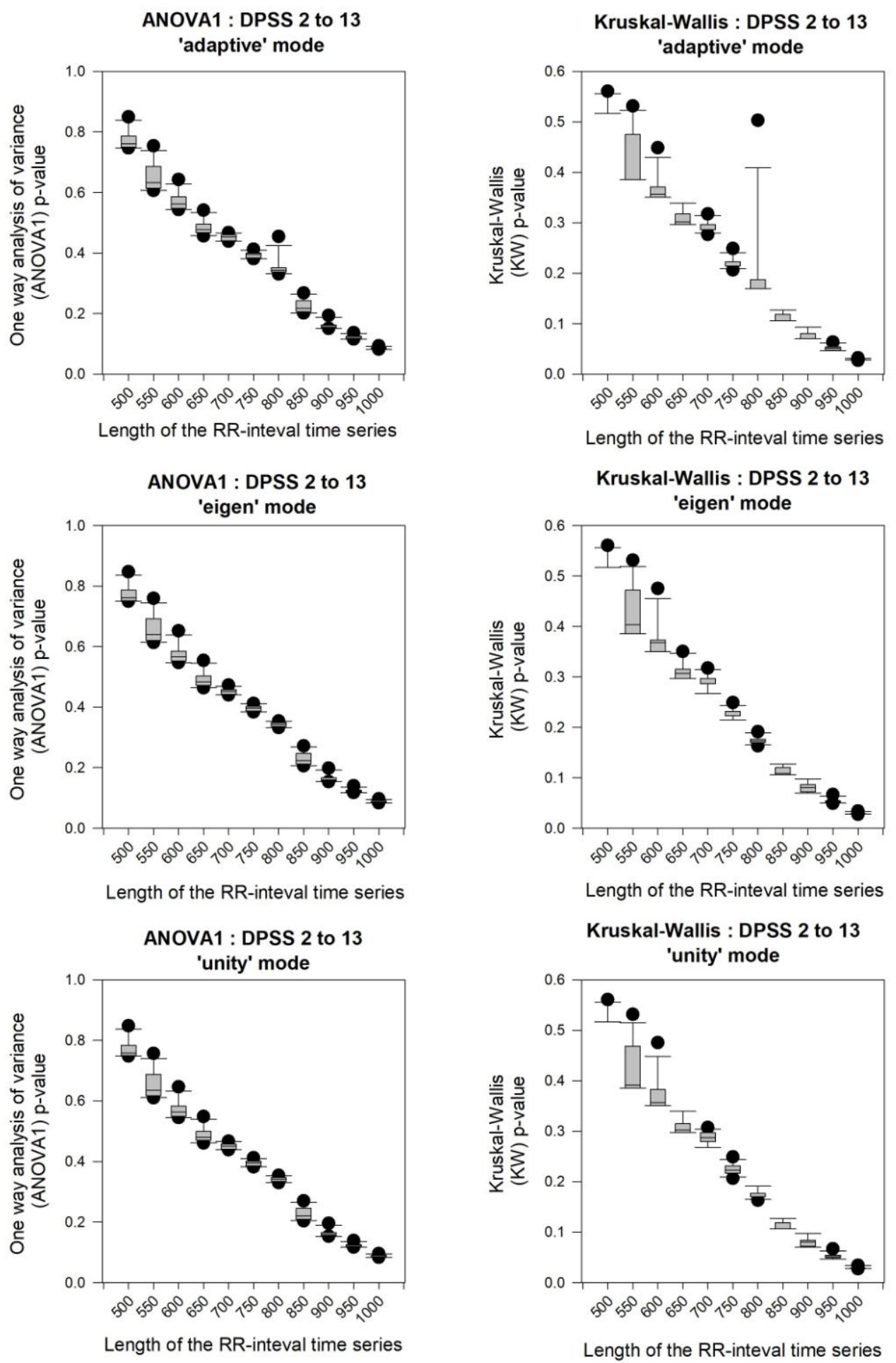

Figure 3. The boxplots of CFP1 for the two tests of significance, one-way analysis of variance (ANOVA1: left column) and the Kruskal-Wallis test (right column) by p-value for the normal against diabetes mellitus subjects. These are per DPSS values from 2 to 13 in increments of one and for length of time-series from 500 to 1000 RR-intervals in intervals of 50. The three Thomson's nonlinear combinations are applied: 'adaptive' (top row), 'eigen' (middle row) and 'unity' (bottom row). The value closest to zero is the minimum with the value furthest away the maximum. The boundary of the box nearby the zero specifies the $25^{\text {th }}$ percentile. The line within the box is the median; not the mean. The boundary of the box furthest from the zero is the $75^{\text {th }}$ percentile. The difference flanked by these boundary points is the inter-quartile range (IQR). Whiskers (or error bars) above and beneath the boxes designate the $90^{\text {th }}$ and $10^{\text {th }}$ percentiles respectively. 


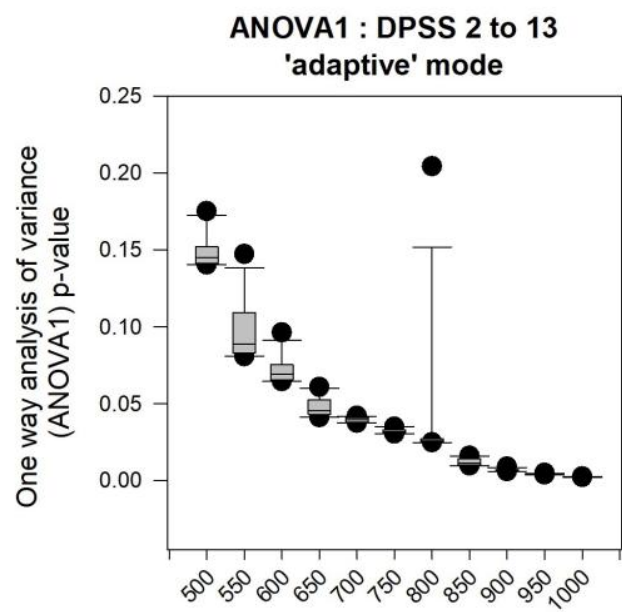

Length of the RR-inteval time series

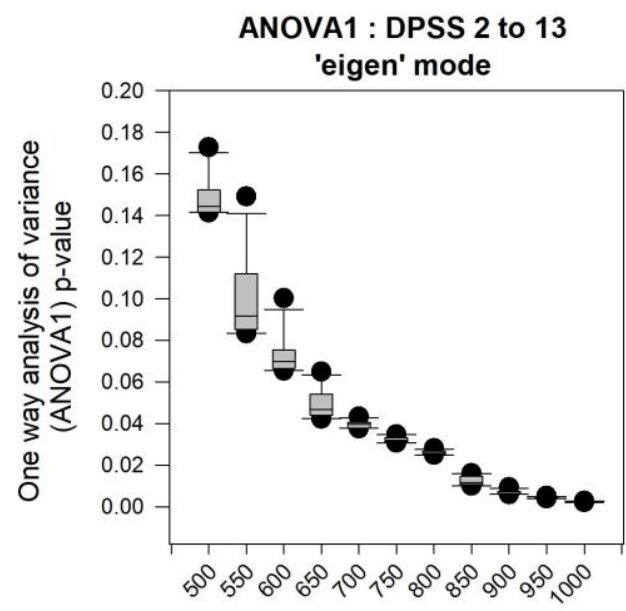

Length of the RR-inteval time series

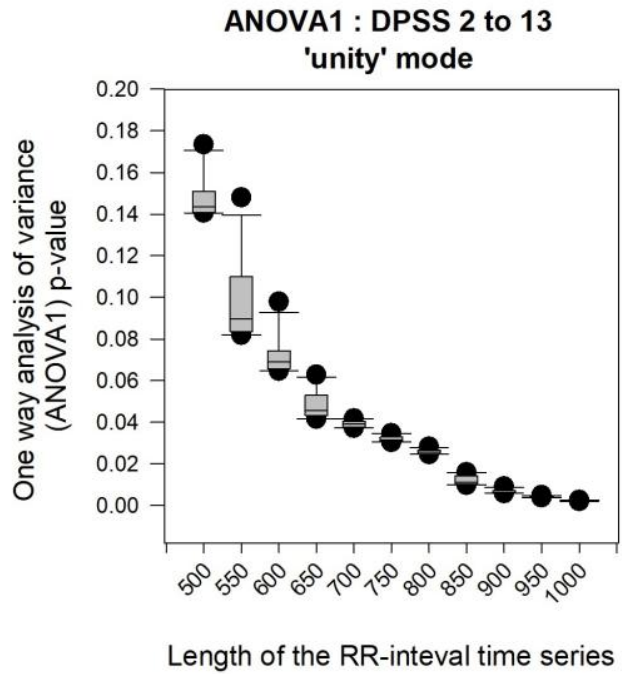

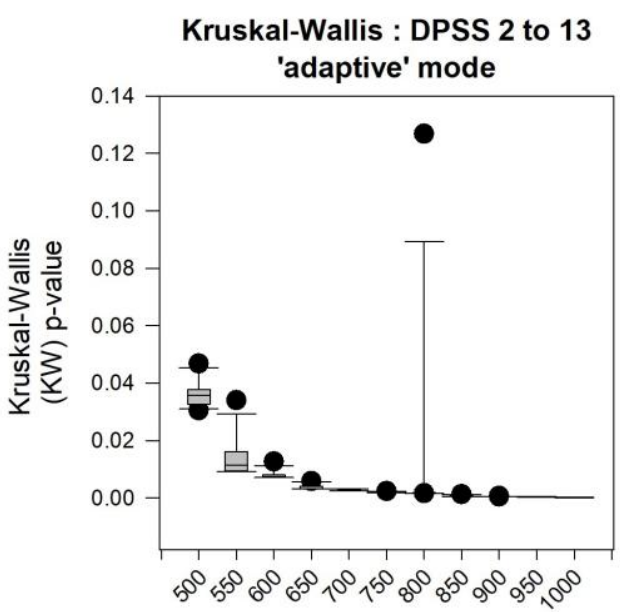

Length of the RR-inteval time series

Kruskal-Wallis : DPSS 2 to 13 'eigen' mode

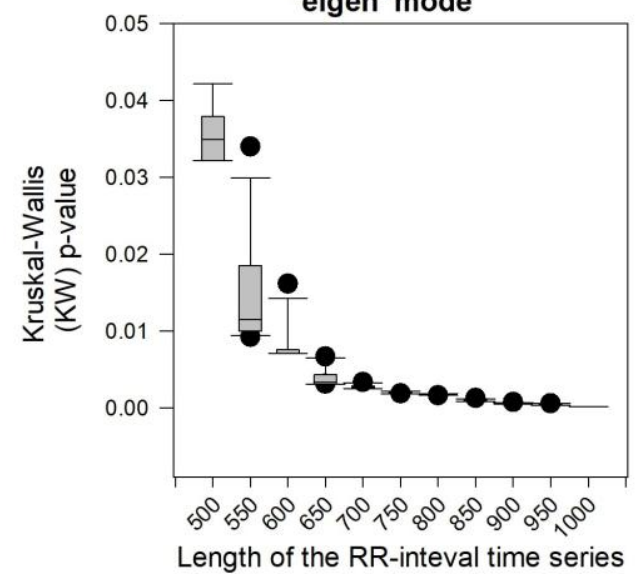

Kruskal-Wallis : DPSS 2 to 13 'unity' mode

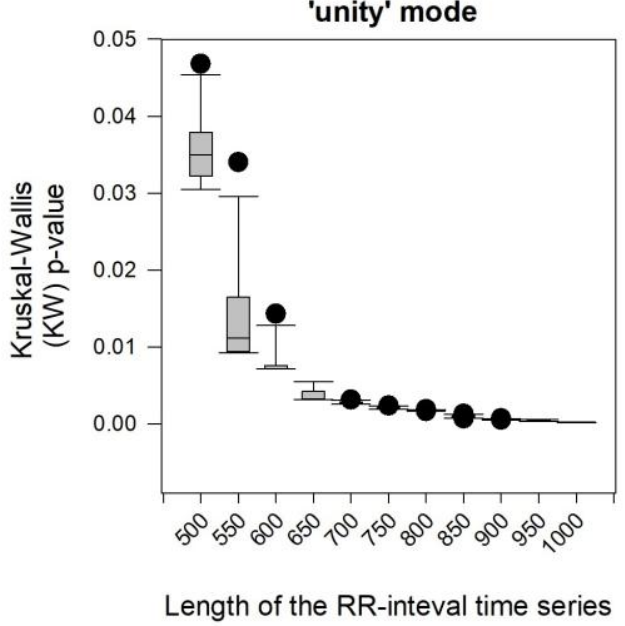

Figure 4. The boxplots of CFP3 for the two tests of significance, one-way analysis of variance (ANOVA1: left side) and the Kruskal-Wallis test (right side) by p-value for the normal versus diabetes mellitus subjects. These are per DPSS values from 2 to 13 in increments of one and for length of time-series from 500 to 1000 RR-intervals in intervals of 50 . The Thomson's nonlinear combinations are applied: 'adaptive' (upper level), 'eigen' (middle level) and 'unity' (lower level). 
Table 2. CFP1 and CFP3 (non-dimensional values) for the two tests of significance; the ANOVA1 (parametric) and the Kruskal-Wallis (non-parametric) test by p-value for the normal against diabetic subjects. DPSS is set to 3 and length of time-series is set at $1000 \mathrm{RR}$-intervals for an interpolation rate of $1 \mathrm{~Hz}$ increasing to $2000 \mathrm{for} 2 \mathrm{~Hz}$ and so on. To calculate the MTM, the additional settings are as follows, sampling frequency of 1Hz, 256 for Fast Fourier Transform length and

Thomson's 'adaptive' nonlinear combination method.

\begin{tabular}{|l|l|l|l|l|}
\hline \multirow{2}{*}{$\begin{array}{l}\text { Interpolation } \\
\text { Rate }(\mathrm{Hz})\end{array}$} & $\begin{array}{l}\text { CFP1 } \\
(\mathrm{p} \text {-value })\end{array}$ & $\begin{array}{l}\text { CFP3 } \\
(\mathrm{p} \text {-value })\end{array}$ \\
\cline { 2 - 5 } & ANOVA1 & KW & ANOVA1 & KW \\
\hline 1 & 0.0888 & 0.0273 & 0.0024 & 0.0002 \\
\hline 2 & 0.0914 & 0.0273 & 0.0024 & 0.0002 \\
\hline 3 & 0.0924 & 0.0273 & 0.0024 & 0.0002 \\
\hline 4 & 0.0928 & 0.0273 & 0.0024 & 0.0002 \\
\hline 5 & 0.0932 & 0.0273 & 0.0024 & 0.0002 \\
\hline 6 & 0.0937 & 0.0273 & 0.0024 & 0.0002 \\
\hline 7 & 0.0934 & 0.0288 & 0.0024 & 0.0002 \\
\hline 8 & 0.0940 & 0.0273 & 0.0024 & 0.0002 \\
\hline 9 & 0.0939 & 0.0288 & 0.0024 & 0.0002 \\
\hline 10 & 0.0941 & 0.0305 & 0.0024 & 0.0002 \\
\hline 11 & 0.0941 & 0.0288 & 0.0024 & 0.0002 \\
\hline 12 & 0.0945 & 0.0273 & 0.0024 & 0.0002 \\
\hline 13 & 0.0948 & 0.0305 & 0.0024 & 0.0002 \\
\hline
\end{tabular}

Reduced complexity of HRV is associated with impaired autonomic function $[\underline{45}, \underline{46}]$. It allowed us to hypothesize decreased values of globally chaotic parameters in diabetic patients, which was later confirmed by lower values of CFP1 and CFP3 in the diabetic group compared to the control group.

Regarding the mathematical particulars of the globally chaotic methods, in this study we intended to maximize the significance of the discrimination between the two cohorts by adjusting the parameters of MTM power spectrum applied in the calculation of chaotic globals. CFP1 and CFP3 were the only significant combinations of chaotic globals when assessed by the MTM power spectrum and the combinations of $h s$ Entropy, $h s$ DFA and sMTM under default conditions. Therefore, when we adjusted the MTM parameters we examined the effects of adjustment on these two combinations alone. The three Thomson's nonlinear combination methods ('adapt', 'eigen' and 'unity') have similar effect throughout and indicate no difference in the significances when applied sequentially. DPSS was varied between 2 to 13 . Yet, these adjustments had little significance on the outcome of the statistical tests (ANOVA1 or Kruskal-Wallis), except for minimal influence when the data lengths were extremely short (RR-intervals < 600). The majority of the differences were produced by signal length.

Furthermore, once we had adjusted the MTM power spectrum parameters we assessed the impact of a cubic spline interpolation on the time series. Interpolation of the RR-intervals time-series prior to enforcement of the MTM power spectra was irrelevant. It had little effect on the statistical significance between the two groups.

Globally chaotic methods applied to RRintervals were previously evaluated in ADHD [15], COPD [14], Obesity [흐, $\underline{47]}$, Malnutrition [48] and flexible pole manuevers for physiotherapy shoulder rehabilitation [49]. This study highlights significant findings for clinical practice and procedures. ICUs and physicians are interested in predicting the risk for physiological complications. Comprehension of biological signals through nonlinear HRV is a significant issue for an appropriate program of care. We revealed that globally chaotic methods 
applied to RR-intervals is sensitive to distinguish autonomic impairment in diabetes. Yet, they are robust to MTM spectral parameters and cubic spline interpolations.

\section{Conclusions}

Chaotic global techniques applied to RRintervals robustly detected HRV changes in diabetic patients revealing decreased nonlinear mechanisms in this population. Spectral adjustments or interpolation of time-series induced only slight effects. Therefore, this technique was able to identify autonomic dysfunction in diabetes mellitus.

Acknowledgements \& Duality of Interest. The authors declare that there are no conflict of interests concerning the publication of this article. Financial funding was provided by $\mathrm{CNPq}$ - number process: 477442/2012-9 and FAPESP

\section{REFERENCES}

1. Campos LA, Pereira Jr VL, Muralikrishna A, Albarwani S, Brás S, Gouveia S. Mathematical biomarkers for the autonomic regulation of cardiovascular system, Frontiers in physiology 4 279, 2013.

2. Goldberger AL, Cardiac chaos. Science 243: 1419, 1989.

3. Goldberger AL, West BJ. Chaos and order in the human body. MD Comput 9: 25-34, 1992.

4. Garner DM, De Souza NM, Vanderlei LCM. Risk Assessment of Diabetes Mellitus by Chaotic Globals to Heart Rate Variability via Six Power Spectra. Rom J Diab Metab Dis 24(3): 227-236, 2017.

5. Seiver A, Daane S, Kim R. Regular low frequency cardiac output oscillations observed in critically ill surgical patients. Complexity 2: 51-55, 1997.

6. Kawaguchi M, Takamatsu I, Kazama $\mathbf{T}$. Rocuronium dose-dependently suppresses the spectral entropy response to tracheal intubation during propofol anaesthesia. Br J Anaesth 102: 667-672, 2009.

7. Alvarez D, Hornero R, Marcos J, Del Campo F, Lopez M. Spectral analysis of electroencephalogram and oximetric signals in obstructive sleep apnea diagnosis, Conf Proc IEEE Eng Med Biol Soc 2009: 400-403, 2009.

8. Schmidt M, Banzett RB, Raux $M$ et al. Unrecognized suffering in the ICU: addressing dyspnea in mechanically ventilated patients. Intensive Care Med 40: $1-10,2014$.

9. Banzett RB, Lansing $\mathbf{R W}$, Brown $\mathbf{R}$ et al. 'Air hunger' from increased $\mathrm{PCO} 2$ persists after complete neuromuscular block in humans. Respir Physiol 81: 1-17, 1990 .
10. Goldberger AL, Rigney DR, Mietus J, Antman EM, Greenwald S. Nonlinear dynamics in sudden cardiac death syndrome: heartrate oscillations and bifurcations. Experientia 44: 983-987, 1988.

11. Goldberger AL, Peng CK, Lipsitz LA. What is physiologic complexity and how does it change with aging and disease? Neurobiol Aging 23: 23-26, 2002.

12. Ponnusamy A, Marques JL, Reuber $M$. Comparison of heart rate variability parameters during complex partial seizures and psychogenic nonepileptic seizures. Epilepsia 53: 1314-1321, 2012.

13. Ponnusamy A, Marques JL, Reuber M. Heart rate variability measures as biomarkers in patients with psychogenic nonepileptic seizures: potential and limitations. Epilepsy Behav 22: 685-691, 2011.

14. Bernardo AFB, Vanderlei LCM and Garner DM. HRV Analysis - A clinical and diagnostic tool in Chronic Obstructive Pulmonary Disease, International Scholarly Research Notices 2014: 1-6, 2014.

15. Wajnsztejn R, De Carvalho TD, Garner DM et al. Heart rate variability analysis by chaotic global techniques in children with attention deficit hyperactivity disorder. Complexity 21: 412-419, 2016.

16. Hall JE. Guyton and Hall textbook of medical physiology. Elsevier Health Sciences, 2010.

17. Wiertel-Krawczuk A, Hirschfeld AS, Huber J, Wojtysiak M, Szymankiewicz-Szukala A. Sympathetic skin response following single and combined sound and electrical stimuli in young healthy subjects. J Med Sci 85: 106-113, 2016.

18. Baum P, Petroff $D$, Classen J, Kiess W, Bluher S. Dysfunction of autonomic nervous system in childhood 
obesity: a cross-sectional study. PLoS One 8: e54546, 2013.

19. Mackey MC, Milton JG. Dynamical diseases. Ann N Y Acad Sci 504: 16-32, 1987.

20. Welch $\mathbf{P}$. The use of fast Fourier transform for the estimation of power spectra: a method based on time averaging over short, modified periodograms. IEEE Transactions on audio and electroacoustics 15: 70-73, 1967.

21. Oppenheim AV, Schafer RW, Buck JR Discrete-time signal processing. 5th ed. Prentice Hall Upper Saddle River, 1999.

22. Souza NM, Vanderlei LC, Garner DM. Risk evaluation of diabetes mellitus by relation of chaotic globals to HRV. Complexity 20: 84-92, 2015.

23. Tarvainen MP, Niskanen J-P, Lipponen JA, Ranta-Aho PO, Karjalainen PA. Kubios HRV-heart rate variability analysis software. Comput Methods Program Biomed 113: 210-220, 2014.

24. Sassi R, Cerutti S, Lombardi F et al. Advances in heart rate variability signal analysis: joint position statement by the e-Cardiology ESC Working Group and the European Heart Rhythm Association co-endorsed by the Asia Pacific Heart Rhythm Society. Ep Europace 17: 1341-1353, 2015.

25. Slepian S. Prolate spheroidal wave functions, Fourier analysis and uncertainty-V, The discrete case. Bell Syst Tech J 57: 1371-1430, 1978.

26. Shannon CE. A Mathematical Theory of Communication. The Bell System Technical Journal 27: 379-423, 1948.

27. Peng CK, Havlin S, Stanley HE, Goldberger AL. Quantification of scaling exponents and crossover phenomena in nonstationary heartbeat time series. Chaos 5: 82-87, 1995.

28. Garner DM, Ling BWK. Measuring and locating zones of chaos and irregularity. J Syst Sci Complex 27: 494-506, 2014.

29. Jolliffe IT. Principal Component Analysis, Series: Springer Series in Statistics. 2nd ed., Springer: New York, 2002.

30. Manly BF. Multivariate statistical methods: a primer: CRC Press, 2004.
31. Sullivan GM, Feinn R. Using effect size - or why the $\mathrm{P}$ value is not enough, $J$ Grad Med Educ 4(3): 279$282,2012$.

32. Coe R. It's the effect size, stupid: What effect size is and why it is important. Annual Conference of the British Educational Research Association, 2002.

33. Lakens D. Calculating and reporting effect sizes to facilitate cumulative science: a practical primer for $\mathrm{t}$ tests and ANOVAs. Frontiers in psychology 4: 863, 2013.

34. Sawilowsky SS. New effect size rules of thumb, 2009.

35. Baselli G, Cerutti S, Civardi $\mathbf{S}$ at al. Heart rate variability signal processing: a quantitative approach as an aid to diagnosis in cardiovascular pathologies. Int $J$ Biomed Comput 20(1-2): 51-70, 1987.

36. Vanderlei FM, Vanderlei LC, Garner DM. Chaotic global parameters correlation with heart rate variability in obese children. J Hum Growth Dev 24(1): 24-30, 2014.

37. Mateo J, Laguna $\mathbf{P}$. Improved heart rate variability signal analysis from the beat occurrence times according to the IPFM model. IEEE Transactions on Biomedical Engineering 47(8): 985-996, 2000.

38. DeBoer RW, Karemaker JM, Strackee J. Comparing spectra of a series of point events particularly for heart rate variability data. IEEE Transactions on Biomedical Engineering 31(4): 384-387, 1984.

39. Kreyszig E. Advanced engineering mathematics. Wiley, 2011.

40. Camm AJ, Malik M, Bigger JT et al. Heart rate variability: standards of measurement, physiological interpretation and clinical use. Task Force of the European Society of Cardiology and the North American Society of Pacing and Electrophysiology. Circulation 93: 1043-1065, 1996.

41. Anderson TW, Darling DA. A test of goodness of fit. Journal of the American Statistical Association 49(268): 765-769, 1954.

42. Razali NM, Wah YB. Power comparisons of shapiro-wilk, kolmogorov-smirnov, lilliefors and anderson-darling tests. Journal of Statistical Modeling and Analytics 2(1): 21-33, 2011.

43. Bissinger A. Cardiac Autonomic Neuropathy: Why Should Cardiologists Care about That? J Diabetes Res 2017, 2017. 
44. Fisher VL, Tahrani AA Cardiac autonomic neuropathy in patients with diabetes mellitus: current perspectives. Diabetes Metab Syndr Obes 10: 419, 2017.

45. Costa MD, Davis RB, Goldberger AL. Heart Rate Fragmentation: A Symbolic Dynamical Approach. Front Physiol 8: 827, 2017.

46. Souza NM, Giacon TR, Pacagnelli FL, Barbosa MP, Valenti VE, Vanderlei LC. Dynamics of heart rate variability analysed through nonlinear and linear dynamics is already impaired in young type 1 diabetic subjects. Cardiol Young 26(7): 1383-1390, 2016.
47. Vanderlei FM, Vanderlei LCM, Garner DM. Heart rate dynamics by novel chaotic globals to HRV in obese youths. J Hum Growth Dev 25(1): 82-88, 2015.

48. Barreto GS, Vanderlei FM, Vanderlei LCM, Garner DM. Risk appraisal by novel chaotic globals to HRV in subjects with malnutrition. J Hum Growth Dev 24: 243-248, 2014

49. Antonio AMS, Garner DM, Cardoso MA et al. Behaviour of globally chaotic parameters of heart rate variability following a protocol of exercise with flexible pole. Russ J Cardiol 4: 24-28, 2015. 